\title{
Barbara Stollberg-Rilinger, Maria Theresia, die Kaiserin in ihrer Zeit. Eine Biographie
}

\section{Claire Gantet}

\section{OpenEdition}

\section{Journals}

Édition électronique

URL : http://journals.openedition.org/ifha/9282

DOI : 10.4000/ifha.9282

ISSN : 2198-8943

\section{Éditeur}

IFRA - Institut franco-allemand (sciences historiques et sociales)

\section{Référence électronique}

Claire Gantet, «Barbara Stollberg-Rilinger, Maria Theresia, die Kaiserin in ihrer Zeit. Eine Biographie », Revue de l'IFHA [En ligne], Date de recension, mis en ligne le 20 juin 2018, consulté le 24 septembre 2020. URL : http://journals.openedition.org/ifha/9282 ; DOI : https://doi.org/10.4000/ifha.9282

Ce document a été généré automatiquement le 24 septembre 2020

(CIFHA 
Barbara Stollberg-Rilinger, Maria Theresia, die Kaiserin in ihrer Zeit. Eine Biographie

Claire Gantet

\section{RÉFÉRENCE}

Barbara Stollberg-Rilinger, Maria Theresia, die Kaiserin in ihrer Zeit. Eine Biographie, 2.

Aufl., München: Beck, 2017, XXVIII-1083 p., $34 €$ 
Aussitôt célébrée comme un chefd'œuvre, honoré dès sa parution par le prix de la Foire du livre de Leipzig en 2017 et réimprimé après quelques mois, la biographie de l'impératrice Marie-Thérèse par Barbara Stollberg-Rilinger suscite de fortes attentes. Elle est plus que le fruit du hasard conjoncturel de la commémoration des 300 ans de la naissance de l'impératrice le 13 mai 1717. Face à une historiographie «monumentale»-dont le sommet fut l'Histoire de Marie-Thérèse (Geschichte Maria Theresias) d'Alfred von Arneth en dix volumes parue entre 1863 et 1879 -, et face à une historiographie masculine qui thématisa l'impératrice en une héroïne, mère féconde, régente et pieuse - une sorte de femme au foyer d'Empire ( Reichshausfrau ", p. XXIII) -, B. Stollberg-Rilinger entend

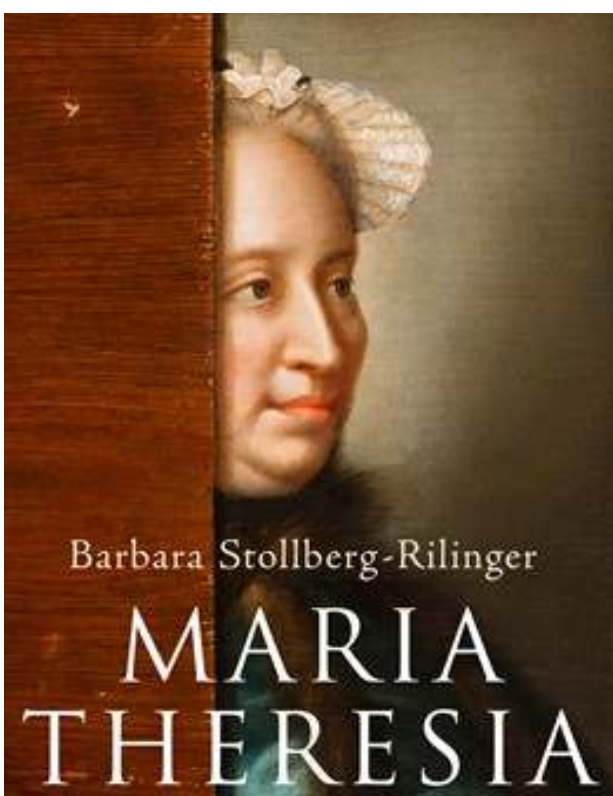

DIE KAISERIN IN IHRER ZEIT Eine Biographie C.H.Beck ne pas soumettre le personnage de Marie-

Thérèse à des normes étrangères à son temps, mais reconstruire l'étrangeté de l'habitus (un terme fréquemment employé), des codes et des croyances qui animèrent son action, et en livrer une biographie ouverte, combinant le récit et l'analyse, non pas l'empathie (un terme dont l'auteur s'abstient) mais la proximité et la mise en perspective. Ce livre vient ainsi combler l'absence d'une biographie récente de l'impératrice. Ce qu'il ne thématise pas, en revanche, est le renouveau du genre de la biographie et des études sur les cultures auliques, dans lequel il s'insère et qui contribue à son succès. L'angle d'approche retenu pour le fil biographique est l'histoire dynastique. Fondé sur une importante documentation de première main (archives manuscrites et ouvrages imprimés antérieurs à 1800), ce livre nous fait pénétrer dans la cour de Vienne en la période charnière du milieu du XVIII ${ }^{e}$ siècle: dans les jeux d'étiquette et de protocole, dans les relations de clientélisme et les fantasmes de contrôle, dans le rapport aux corps, à l'éducation et à la sexualité. Son récit procède de façon globalement chronologique, avec quelques arrêts sur image.

Les premiers chapitres, qui portent sur la jeunesse de Marie-Thérèse jusqu'au décès de son père Charles VI alors qu'elle avait 23 ans, nous proposent une nouvelle pondération d'un fil globalement déjà connu: si Marie-Thérèse n'a certes pas étudié le droit (la « jurisprudence »), elle a néanmoins reçu une éducation conforme à celle des archiducs. Héritière habsbourgeoise potentielle en vertu de la Pragmatique Sanction de 1713 qui déclarait indivisibles les États patrimoniaux, elle n'a toutefois pas pu travailler dans la Conférence secrète, fréquentée par des hommes. Sa position à la cour est surtout érodée par son mariage en 1736 avec François-Étienne de Lorraine, qu'elle connait et estime mais qui relève d'une noblesse peu illustre en proie aux puissants (il dut renoncer à la Lorraine contre l'expectative de la Toscane) et ne s'est pas illustré par la gloire militaire. Si l'union est heureuse, si Marie-Thérèse lui est toujours restée fidèle (ce que François-Étienne ne lui a sans doute pas toujours rendu, p. 276), FrançoisÉtienne devient rapidement la cible de propos dénonçant son irrésolution, sa faiblesse 
et son manque d'ardeur. Le couple a seize enfants, dont dix atteignent l'âge adulte, et fonctionne sur un mode complémentaire : là où son mari est porté à la tempérance et à la modestie, Marie-Thérèse a un caractère fougueux, sûr, apte à s'imposer. À la mort de Charles VI, Marie-Thérèse est non sans difficultés proclamée «roi » de Hongrie et de Bohême, dans le contexte de la (première) guerre de Silésie puis de la guerre de Succession d'Autriche. Dans un bref chapitre (p. 145-176), B. Stollberg-Rilinger nous rappelle que Marie-Thérèse a délibérément renoncé à un couronnement en tant qu'impératrice mais fait couronner son mari en 1745 : le titre d'impératrice ne lui est revenu qu'en tant qu'épouse de l'empereur (p. 148). Pour elle, l'essentiel est en effet les États patrimoniaux : la "politique impériale » est une "politique domestique avec des moyens impériaux, non une politique orientée vers l'Empire en tant qu'entité politique » (p. 176). B. Stollberg-Rilinger nous montre que François-Étienne a pris part au gouvernement et régulièrement participé avec sa femme à la Conférence aulique, où il a souvent soutenu des opinions contraires, notamment à celle de Kaunitz, le conseiller promu par Marie-Thérèse, et quant aux relations avec la Prusse.

L'historiographie a longtemps célébré les réformes étatiques introduites par MarieThérèse, et plus récemment réinterprété les réformes de son fils Joseph II dans leur lignée. B. Stollberg-Rilinger leur consacre peu de lignes, en argumentant qu'elles n'ont pas entraîné les effets désirés, et s'attache plus aux relations de plus en plus difficiles avec la noblesse. Elle nous plonge ensuite dans les codes corporels et érotiques de l'aristocratie (p. 247-316), en une période de basculement d'un habitus très libéral (du moins pour ce qui concerne les hommes, et bientôt taxé de galanterie française) vers la sensiblerie (rapidement présentée comme bourgeoise-allemande). Marie-Thérèse frappe par sa rigidité à partir de 1747 et n'hésite pas à placer ses «mouches » pour parer aux déviances, avec un résultat peu probant. Elle nomme un médecin lettré, Gerard Van Swieten, comme médecin personnel, lequel surveille ses grossesses et supervise les sages-femmes. L'étude du monde aulique se poursuit par les « distinctions et finesses» (p. 317-399) : la recherche d'une distance face à la bourgeoisie lors de la pratique des suppliques ou de tous les rites de passage, l'éclat de la cour étant adressé avant tout à la noblesse; les habits à l'espagnole contre le «diktat» de la mode française (du moins jusqu'aux nouveautés de Kaunitz), les fêtes qui se heurtent là aussi à un désintérêt croissant de la noblesse pour une vie trop coûteuse et aspire de plus en plus au naturel (p. 399).

B. Stollberg-Rilinger consacre peu de pages au «renversement des alliances » bien connu de 1756 et rappelle les tentatives menées par Frédéric In pour styliser la guerre de Sept ans (1756-1763) en une guerre de religion ainsi que l'action des propagandes et de l'espionnage durant une guerre "désastreuse » pour Marie-Thérèse (p. 401-459). Elle s'attarde plus sur le «capital de la dynastie » (p. 461-515), c'est-à-dire sur l'éducation des enfants de Marie-Thérèse et à sa politique matrimoniale. Elle signale au passage les relations homosexuelles de Marie-Christine et de l'intelligente et charmante Isabelle de Parme (la première épouse de Joseph et la seule qu'il aima). Elle se penche ensuite sur l'action de Van Swieten pour combattre la petite vérole et introduire l'inoculation, adoptée par Marie-Thérèse.

Le tournant de la vie de Marie-Thérèse est le décès de François-Étienne en 1765, dont elle dit ne s'être jamais remise. Désormais, Joseph est sans autre cérémonie empereur puisqu'il a été élu roi des Romains en 1764, mais il n'obtient un territoire propre que lorsque sa mère lui concède le titre très vague et peu confortable de co-régent. En tant 
que fils, il est enfin astreint à l'obéissance vis-à-vis de Marie-Thérèse. Les conflits apparaissent dès le deuil, lorsque Joseph II opte pour l'uniforme militaire, critique Kaunitz, réforme l'armée sur le modèle prussien honni par sa mère, accepte rapidement (cette fois avec Kaunitz) le (premier) partage de la Pologne, adopte des mœurs sexuelles laxistes et prône la tolérance religieuse là où sa mère, bien que catholique pragmatique (notamment dans ses choix entre jésuites et jansénistes, p. 575-627), poursuit tous les courants distincts de la pietas austriaca (p. 629-679). Dans ses relations avec ses sujets (p.629-679), Marie-Thérèse représente là aussi un paternalisme - ou maternalisme (p. 683) - en partie déjà désuet. Elle aspire certes à une information exhaustive, organisant à cette fin une Seelenkonskription en 1770-1771, s'alarme de la situation en Bohême, propose des réformes plus radicales que son fils quant à l'affranchissement des paysans et impose une réforme fondamentale des écoles primaires (p. 714), mais son code pénal de 1769, avec ses dispositions sur la torture et les peines corporelles relève du passé. Lors de la guerre de Succession de Bavière, les dissensions entre la mère et le fils sont patentes, publiques.

Le dernier chapitre (p. 753-833) est consacré aux échecs de Marie-Thérèse à imposer une ligne politique conforme à ses enfants installés en France (Marie-Antoinette) ou plus encore en Italie (Marie-Amélie), aux relations parfois de haine entre frères et sœurs, et à la stylisation de sa propre mort. Contre l'historiographie «monumentale ", l'épilogue (p. 835-855) avance un jugement sévère: Marie-Thérèse a agi telle une " araignée dans sa toile de communication » hantée par des "fantasmes de contrôle " (p. 847); «sa tragédie a consisté à camper sur des normes qui n'étaient plus guère partagées et à vue d'œil condamnées à l'échec » (p. 855).

Par son ampleur, sa densité et son écriture, par la nouveauté de certains apports, cette biographie s'impose comme un ouvrage de référence incontournable. Comme tout livre toutefois, il suscite des questions, qui ont trait non aux analyses précises, toujours clairvoyantes et probantes, mais au cadre d'interprétation général. Certes, B. StollbergRilinger lutte contre l'historiographie dite monumentale. La perspective centrée sur la personne de Marie-Thérèse insérée dans une culture aulique, et non sur la monarchie des Habsbourg ou sur les relations à l'Empire ne conduit-elle pas l'auteur à gauchir un jugement sévère en offrant finalement un récit fermé ? Ne force-t-elle pas le trait lorsqu'elle écrit qu'en 1745, Marie-Thérèse « dédaigne les formalités ancestrales du Saint-Empire " et quand elle qualifie "la dignité impériale d'honneur vide et le couronnement de comédie» (p. 149) ? L'histoire du Saint-Empire au XVIII ${ }^{\mathrm{e}}$ siècle se réduit-elle à un lent déclin déterminé par une incapacité à moderniser des structures féodales? Une certaine politisation n'a-t-elle pas eu lieu dans le cadre féodal?

\section{INDEX}

Index chronologique : Période moderne

Thèmes : Histoire des États et des pouvoirs, Histoire des femmes/histoire du genre 
AUTEURS

CLAIRE GANTET

Université Fribourg (Suisse)/Universität Freiburg (Schweiz) 